\title{
Labeled Factorization of Integers
}

\author{
Augustine O. Munagi \\ John Knopfmacher Centre for Applicable Analysis and Number Theory \\ School of Mathematics, University of the Witwatersrand \\ Wits 2050, Johannesburg, South Africa \\ Augustine.Munagi@wits.ac.za
}

Submitted: Jan 5, 2009; Accepted: Apr 16, 2009; Published: Apr 22, 2009

Mathematics Subject Classification: 11Y05, 05A05, 11B73, 11B13

\begin{abstract}
The labeled factorizations of a positive integer $n$ are obtained as a completion of the set of ordered factorizations of $n$. This follows a new technique for generating ordered factorizations found by extending a method for unordered factorizations that relies on partitioning the multiset of prime factors of $n$. Our results include explicit enumeration formulas and some combinatorial identities. It is proved that labeled factorizations of $n$ are equinumerous with the systems of complementing subsets of $\{0,1, \ldots, n-1\}$. We also give a new combinatorial interpretation of a class of generalized Stirling numbers.
\end{abstract}

\section{Ordered and labeled factorization}

An ordered factorization of a positive integer $n$ is a representation of $n$ as an ordered product of integers, each factor greater than 1 . The set of ordered factorizations of $n$ will be denoted by $F(n)$, and $|F(n)|=f(n)$. For example, $F(6)=\{6,2.3,3.2\}$. So $f(6)=3$.

Every integer $n>1$ has a canonical factorization into prime numbers $p_{1}, p_{2}, \ldots$, namely

$$
n=p_{1}^{m_{1}} p_{2}^{m_{2}} \ldots p_{r}^{m_{r}}, \quad p_{1}<p_{2}<\cdots<p_{r}, m_{i}>0,1 \leq i \leq r .
$$

The enumeration function $f(n)$ does not depend on the size of $n$ but on the exponents $m_{i}$. In particular we define

$$
\Omega(n)=m_{1}+m_{2}+\cdots+m_{r}, \Omega(1)=0 .
$$

Note that the form of (1) may sometimes suggest a formula for $f(n)$. For instance,

- $n=p^{m}$ gives $f(n)=2^{m-1}$, the number of compositions of $m$. 
- $n=p_{1} p_{2} \ldots p_{r}$ gives $f(n)=\sum_{k=1}^{r} k ! S(r, k)$, the $r^{t h}$ ordered Bell number; $S(n, k)$ is the Stirling number of the second kind.

A general formula for the number $f(n, k)$ of ordered $k$-factorizations of $n$ was found in 1893 by MacMahon [9] (also [1, p. 59]):

$$
f(n, k)=\sum_{i=0}^{k-1}(-1)^{i}\left(\begin{array}{l}
k \\
i
\end{array}\right) \prod_{j=1}^{r}\left(\begin{array}{c}
m_{j}+k-i-1 \\
m_{j}
\end{array}\right) .
$$

Thus $f(n)=f(n, 1)+f(n, 2)+\cdots+f(n, \Omega(n))$.

It will be useful to review some techniques for generating ordered factorizations. The simplest approach is perhaps to obtain the set of unordered factorizations of $n$, and replace each member by the permutations of its factors. Another method is provided by the classical recurrence relation

$$
f(1)=1, \quad f(n)=\sum_{\substack{d \mid n \\ d<n}} f(d)
$$

If a positive integer $d$ divides $n$, denoted by $d \mid n$, then each element of $F(d), d<n$, gives a unique element of $F(n)$ by appending $n / d$. Thus if the proper divisors of $n$ are $d_{1}, d_{2}, \ldots, d_{\tau(n)-1}$, then $F(n)$ is given by

$$
F(n)=F\left(d_{1}\right)\left(n / d_{1}\right) \cup F\left(d_{2}\right)\left(n / d_{2}\right) \cup \ldots \cup F\left(d_{\tau(n)-1}\right)\left(n / d_{\tau(n)-1}\right),
$$

where $\tau(n)$ is the number of positive integral divisors of $n$, and $S r=\{s . r \mid s \in S\}$.

To motivate the next method, observe that the unordered factorizations of $n$ may be generated from the unique representation (1) of $n$ expressed as a sequence of $\Omega(n)$ factors. Denote this factorization by $\operatorname{can}(n)$. Indeed for each positive integer $k \leq \Omega(n)$, a $k$-factorization is obtained by distributing the factors in can $(n)$ into $k$ identical cells without further restriction, replacing each cell by the product of its members, and arranging the factors in nondecreasing order. Lastly, a set of $k$-factorizations is obtained by deleting repeated factorizations. This procedure will be referred to as the Factor algorithm.

So Factor is tantamount to finding the distinct partitions of the multiset $\left\{p_{1} m_{1}, \ldots, p_{r} m_{r}\right\}$ into $k$ blocks. Consequently the generation of ordered factorizations can be viewed as a process of obtaining the ordered partitions of multisets, i.e., the distribution of objects of arbitrary specification into different cells so that no cell is empty.

The above techniques are known (see [6], [7]), but the following approach seems to be new.

An instructive method of generating ordered factorizations is to iterate Factor by replacing the factorization $\operatorname{can}(n)$ with the set $F P(n)$ of permutations of $\operatorname{can}(n)$. Then we notice that each element of $F(n)$ is obtained by multiplying only adjacent factors in a member of $F P(n)$. This differs fundamentally from the construction of unordered 
factorizations which also employed non-adjacent pairings of factors. The procedure for generating $F(n)$ in this context is OrdFactor, which may be viewed as the union of the applications of Factor to each member of $F P(n)$ with the further restriction that only adjacent factors are merged, but generally distinguishing factorizations that differ in the ordering of their elements.

Thus we are led to the natural question of investigating the set $X(n)$ of groupings involving non-adjacent factors in members of $F P(n)^{1}$. The purpose of this paper is to study the function $f f(n)=f(n)+x(n)$, which counts the full set $F F(n)=F(n) \cup X(n)$, where $x(n)=|X(n)|$.

In this larger set the integers appearing in a factorization will be called atoms. All atoms are now subscript-labeled, but the subscripts may be omitted from consecutively labeled atoms when they are obvious. On the other hand, groupings/cells with a number of labeled atoms will be referred to as factors of $n$. Factors will generally be enclosed in parentheses, with the possible exception of factors having single atoms. So atoms and factors are identical in $F(n)$.

$F F(n)$ will be called the set of labeled factorizations of $n$.

A possible characterization is the following:

(*) A labeled factorization of $n$ corresponds to a partition of the set of elements of the sequence of factors in an ordered prime factorization of $n$ which have been tagged with distinct labels.

The corresponding extension of OrdFactor for generating labeled factorizations is LabFactor. This algorithm uses the following rule for elements of $X(n)$ : any sequence of consecutively labeled atoms occurring in a factor may be replaced by the product of the atoms (followed by a size-preserving, standard, relabeling of surviving labels in the factorization, where the product is assumed to bear the smallest label in the sequence).

Example 1.1. The two levels of ordered factorization are illustrated with $n=12$ and $n=16$, using LabFactor. (It is understood that a.b. $\cdots . z=a_{i} \cdot b_{i+1} \cdot \cdots \cdot z_{k}$ for some integers $i, k, 1 \leq i \leq k$.)

$$
\begin{aligned}
& F P(12)=\{2.2 .3,2.3 .2,3.2 .2\} \\
& \longrightarrow\{(2.2 .3),(2.2) .3,2 .(2.3), 2.2 .3,(2.3 .2),(2.3) .2,2 .(3.2), 2.3 .2,(3.2 .2) \text {, } \\
& \longrightarrow\{12,4.3,2.6,2.2 .3,12,6.2,2.6,2.3 .2,12,6.2,3.4,3.2 .2\} \\
& \longrightarrow\{12,4.3,2.6,2.2 .3,6.2,2.3 .2,3.4,3.2 .2\}=F(12) \\
& \Longrightarrow f(12)=8 \text {, }
\end{aligned}
$$

and

$$
\begin{aligned}
& F P(12)=\left\{2_{1} \cdot 2_{2} \cdot 3_{3}, 2_{1} \cdot 3_{2} \cdot 2_{3}, 3_{1} \cdot 2_{2} \cdot 2_{3}\right\} \longrightarrow\left\{\left(2_{1} \cdot 3_{3}\right) \cdot 2_{2},\left(2_{1} \cdot 2_{3}\right) \cdot 3_{2},\left(3_{1} \cdot 2_{3}\right) \cdot 2_{2}\right\}=X(12) . \\
& \text { Hence } f f(12)=f(12)+x(12)=8+3=11 .
\end{aligned}
$$

\footnotetext{
${ }^{1}$ The non-adjacent groupings will not be replaced by their actual products in general.
} 
Similarly, $F P(16)=\{2.2 .2 .2\}$ gives

$$
F(16)=\{16,2.8,4.4,8.2,2.2 .4,2.4 .2,4.2 .2,2.2 .2 .2\} \quad \Longrightarrow f(16)=8
$$

and

$$
\begin{aligned}
X(16)= & \left\{\left(2_{1} \cdot 2_{3} \cdot 2_{4}\right) \cdot 2_{2},\left(2_{1} \cdot 2_{2} \cdot 2_{4}\right) \cdot 2_{3},\left(2_{1} \cdot 2_{3}\right) \cdot\left(2_{2} \cdot 2_{4}\right),\left(2_{1} \cdot 2_{4}\right) \cdot\left(2_{2} \cdot 2_{3}\right),\right. \\
\left.\left(2_{1} \cdot 2_{3}\right) \cdot 2_{2} \cdot 2_{4},\left(2_{1} \cdot 2_{4}\right) \cdot 2_{2} \cdot 2_{3}, 2_{1} \cdot\left(2_{2} \cdot 2_{4}\right) \cdot 2_{3}\right\} & \\
= & \left\{\left(2_{1} \cdot 4_{3}\right) \cdot 2_{2},\left(4_{1} \cdot 2_{3}\right) \cdot 2_{2},\left(2_{1} \cdot 2_{3}\right) \cdot\left(2_{2} \cdot 2_{4}\right),\left(2_{1} \cdot 2_{3}\right) \cdot 4_{2},\left(2_{1} \cdot 2_{3}\right) \cdot 2_{2} \cdot 2_{4},\right. \\
& \left.\left(2_{1} \cdot 2_{4}\right) \cdot 2_{2} \cdot 2_{3}, 2_{1} \cdot\left(2_{2} \cdot 2_{4}\right) \cdot 2_{3}\right\}
\end{aligned}
$$

Hence $f f(16)=f(16)+x(16)=8+7=15$.

Note that LabFactor does not always return unique elements of $X(n)$. For instance, it gives $\left(2_{1} .3_{3} .5_{4}\right) .2_{2},\left(2_{1} .5_{3} .3_{4}\right) .2_{2} \in X(60)$. However, by the rule for elements of $X(n)$, both factorizations are identical, uniquely with $\left(22_{1} \cdot 15_{3}\right) \cdot 2_{2}$. Concise evolutionary procedures are described in Section 2.

Proposition 1.2. (i) $f f\left(p^{m}\right)=B(m)$, where $B(m)$ is the $m^{\text {th }}$ Bell number.

$$
\text { (ii) } f f\left(p_{1} p_{2} \ldots p_{r}\right)=\sum_{k=1}^{r} k ! S(r, k) B(k-1) \text {. }
$$

Proof. (i) This follows at once from the property $\left(^{*}\right)$. So $x\left(p^{m}\right)=B(m)-2^{m-1}$.

(ii) This result is a special case of Corollary 2.7, below.

In Section 2 we obtain enumeration formulas for $f f(n)$ with some combinatorial identities. This is followed, in Section 3, with a brief discussion of permuted (or "ordered") labeled factorizations. In Section 4 we apply labeled factorizations to the enumeration of systems of complementing subsets of $\{1,2, \ldots, n-1\}$ by giving a bijection. A further application is obtained in Section 5 when the enumeration of a distinguished subset of $X(n)$ leads to a class of generalized Bell numbers. The final section discusses few properties of the corresponding Stirling numbers, to be known as B-Stirling numbers of the second kind. In particular we obtain an explicit connection between the B-Stirling numbers and a class of enumeration functions studied by Carlitz in [5].

We will adopt the notational convention: if $H(n)$ is a subset of $F F(n)$, then $H(n, k)$ is the set of elements of $H(n)$ having $k$ factors (or $k$-factorizations), and the corresponding small letters represent cardinalities of sets: $h(n)=|H(n)|, h(n, k)=|H(n, k)|$.

\section{Enumeration formulas}

$f f(n)$ satisfies an analogous relation to (3).

Theorem 2.1. We have

$$
f f(n)=1+\sum_{\substack{d \mid n \\ 1<d<n}} \sum_{k=1}^{\Omega(d)} k f f(d, k)
$$


Proof. The proof is obtained by extending (4) to account for nonadjacent pairings of atoms. By convention we set $f f(1)=f f(1,1)=1$. If $d \mid n, 1<d<n$, then each $h \in F F(d)$ gives non-overlapping elements of $F F(n)$ in two ways:

(i) by appending $n / d$;

(ii) by inserting $n / d$ (bearing the label $k+1$ ) into each of $k-1$ factors of $h \in F F(d, k)$, excluding the factor whose last atom is labeled $k, k \geq 2$.

The first case gives a total of $f f(d)$, while the second case gives $\sum_{k=2}^{\Omega(d)}(k-1) f f(d, k)$ elements of $F F(n)$. Hence the number of contributions to $f f(n)$ is

$$
f f(d)+\sum_{k=2}^{\Omega(d)}(k-1) f f(d, k)=\sum_{k=1}^{\Omega(d)} k f f(d, k) .
$$

Example 2.2. $F F(16)$ is obtained via the relation of Theorem 2.1 as follows.

$$
\begin{aligned}
F F(16)= & \{1\} 16 \cup\{2\} 8 \cup\{4,2.2\} 4 \cup\left\{8,2.4,4.2,\left(2_{1} .2_{3}\right) .2_{2}, 2.2 .2\right\} 2 \\
= & \{16\} \cup\{2.8\} \cup\left\{4.4,2.2 .4,\left(2_{1} .4_{3}\right) .2_{2}\right\} \cup\left\{8.2,2.4 .2,\left(2_{1} .2_{3}\right) .4_{2}, 4.2 .2,\right. \\
& \left.\left(4_{1} .2_{3}\right) .2_{2},\left(2_{1} .2_{3}\right) .2_{2} .2_{4},\left(2_{1} .2_{3}\right) .\left(2_{2} .2_{4}\right), 2.2 .2 .2,\left(2_{1} .2_{4}\right) .2_{2} .2_{3}, 2_{1} .\left(2_{2} .2_{4}\right) .2_{3}\right\} .
\end{aligned}
$$

Remark 2.3. Theorem 2.1 gives $f f\left(p^{m}\right)=1+\sum_{t=1}^{m-1} \sum_{k=1}^{t} k f f\left(p^{t}, k\right)$. Thus, with the formula $f f\left(p^{t}, k\right)=S(t, k)$ and Proposition 1.2(i), we obtain the following identity for the Bell numbers:

$$
B(m)=1+\sum_{t=1}^{m-1} \sum_{k=1}^{t} k S(t, k) .
$$

A direct proof follows by using the standard recurrence

$$
S(n, k)=S(n-1, k-1)+k S(n-1, k), S(0,0)=1, S(1,0)=0,
$$

to show that $\sum_{t=1}^{m-1} \sum_{k=1}^{t} k S(t, k)=\sum_{t=1}^{m-1}(B(t+1)-B(t))$, which telescopes to $B(m)-B(1)$.

Following Example 1.1, we note that $X(n)$ can also be obtained from $F(n)$; after all $F P(n) \subseteq F(n)$. Indeed a moment's reflection shows that each $v \in X(n, k)$ is the result of merging the atoms of a unique $w \in F(n, j), j>k$, into $k$ factors such that only nonadjacent atoms in $w$ belong to a factor (see for example $X(16)$ in Example 1.1 or 2.2)). This observation motivates the following definitions.

Definition 2.4. A factorization $v \in F F(n, k)$ is said to be induced by a partition $\pi$ of $\{1,2, \ldots, j\}$ if $v$ is obtained by merging the atoms of a member of $F(n, j), j \geq k$, so that only atoms bearing the labels in a block of $\pi$ belong to a factor. 
For example, $\left(2_{1} \cdot 4_{3}\right) .2_{2}$ is induced by $\{1,3\}\{2\}$ following operation on $2_{1} \cdot 2_{2} \cdot 4_{3}$.

Definition 2.5. A partition $\pi$ of $\{1,2, \ldots, n\}$ will be called nonadjacent if no block of $\pi$ contains consecutive integers.

Let $\Lambda_{n, k}$ denote the set of nonadjacent partitions of $\{1,2, \ldots, n\}$ into $k$ blocks. The cardinality of $\Lambda_{n, k}$ is known (see Brualdi [3]):

$$
\left|\Lambda_{n, k}\right|=S(n-1, k-1), \quad 1 \leq k \leq n .
$$

Theorem 2.6. We have

$$
f f(1,1)=1, \quad f f(n, k)=\sum_{j=k}^{\Omega(n)} f(n, j) S(j-1, k-1), \quad n \geq 2 .
$$

Proof. As already noted, each $v \in X(n, k)$ is induced by the action of a nonadjacent partition $\pi \in \Lambda(j, k)$ on a factorization $w \in F(n, j), j>k$. Clearly $v$ is uniquely determined by the form of $\pi$ and the ordering of $w$. It follows that for each $k$, the number of contributions to $X(n, k)$ is given exactly by the summation of $|F(n, j)||\Lambda(j, k)|$ over $j, k+1 \leq j \leq \Omega(n)$. Hence we obtain

$$
f f(n, k)=f(n, k)+x(n, k)=f(n, k)+\sum_{j=k+1}^{\Omega(n)} f(n, j)|\Lambda(j, k)|,
$$

which gives the desired result on applying Equation (8).

Corollary 2.7. We have

$$
f f(1)=1, \quad f f(n)=\sum_{j=1}^{\Omega(n)} f(n, j) B(j-1), \quad n \geq 2 .
$$

Remark 2.8. Proposition 1.2( $i)$ can be verified from Corollary 2.7 by using the formula $f\left(p^{m}, j\right)=\left(\begin{array}{c}m-1 \\ j-1\end{array}\right)$ to derive a recurrence relation for the Bell numbers.

Using Theorem 2.1 and Theorem 2.6, we obtain

$$
\sum_{k=1}^{\Omega(d)} k f f(d, k)=\sum_{k=1}^{\Omega(d)} k \sum_{j=k}^{\Omega(d)} f(d, j) S(j-1, k-1)=\sum_{j=1}^{\Omega(d)} f(d, j) \sum_{k=1}^{\Omega(d)} k S(j-1, k-1),
$$

which gives the following identity for any integer $d>0$ :

$$
\sum_{k=1}^{\Omega(d)} k f f(d, k)=\sum_{j=1}^{\Omega(d)} f(d, j) B(j)
$$


Hence we obtain another explicit result for $f f(n)$ :

$$
f f(n)=1+\sum_{\substack{d \mid n \\ d<n}} \sum_{j=1}^{\Omega(d)} f(d, j) B(j)
$$

Note that (9) gives $x(n, k)=\sum_{j=k+1}^{\Omega(n)} f(n, j) S(j-1, k-1)$. Thus with (11), we have two further expressions for $x(n)=f f(n)-f(n)$ :

$$
x(n)=\sum_{j=1}^{\Omega(n)} f(n, j)(B(j-1)-1)=\sum_{\substack{d \mid n \\ d<n}} \sum_{j=1}^{\Omega(d)} f(d, j)(B(j)-1) .
$$

Evaluation of (11) at $n=p^{m}$ gives an iterated recurrence for the Bell numbers:

$$
B(m)=1+\sum_{t=1}^{m-1} \sum_{j=1}^{t}\left(\begin{array}{l}
t-1 \\
j-1
\end{array}\right) B(j) .
$$

\section{Permuted factorizations}

We will call a set $H$ of labeled factorizations permuted if for each $p \in H$, every factorization obtained by permuting the factors of $p$, also belongs to $H$. A bar is placed over each previous notation to distinguish corresponding enumerators of permuted labeled factorizations.

Since the factors of a labeled factorization are distinct (indeed each atom bears a unique label), the number of permuted labeled $k$-factorizations of $n$ is $\overline{f f}(n, k)=$ $k ! f f(n, k)$. Hence the number $\overline{f f}(n)$ of all permuted labeled factorizations of $n$ is given by

$$
\overline{f f}(n)=\sum_{k=1}^{\Omega(n)} \overline{f f}(n, k)=\sum_{k=1}^{\Omega(n)} k ! \sum_{j=k}^{\Omega(n)} f(n, j) S(j-1, k-1) .
$$

That is,

$$
\overline{f f}(n)=\sum_{j=1}^{\Omega(n)} f(n, j) \sum_{k=1}^{j} k ! S(j-1, k-1)
$$

The sum $\sum_{k=1}^{j} k ! S(j-1, k-1)$ is almost an ordered Bell number. So, on using the notation $\sum_{k=1}^{N} k ! S(N, k)=\bar{B}_{N}$, it can be shown that

$$
\sum_{k=1}^{N} k ! S(N-1, k-1)=\frac{1}{2}\left(\bar{B}_{N}+\bar{B}_{N-1}\right), \quad \bar{B}_{0}=1
$$


Thus we have the alternative expression

$$
\overline{f f}(n)=\frac{1}{2} \sum_{j=1}^{\Omega(n)} f(n, j)\left(\bar{B}_{j}+\bar{B}_{j-1}\right) .
$$

Notice that now we have (cf. Proposition 1.2)

Proposition 3.1. (i) $\overline{f f}\left(p^{m}\right)=\bar{B}_{m}$.

(ii) $\overline{f f}\left(p_{1} p_{2} \ldots p_{r}\right)=\frac{1}{2} \sum_{j=1}^{\Omega(r)} j ! S(r, j)\left(\bar{B}_{j}+\bar{B}_{j-1}\right)$

Thus with (13) the following identity holds:

$$
\bar{B}_{m}=\frac{1}{2} \sum_{j=0}^{m-1}\left(\begin{array}{c}
m-1 \\
j
\end{array}\right)\left(\bar{B}_{j}+\bar{B}_{j+1}\right), \quad m>0 .
$$

The enumeration function $\overline{f f}(n)$ gives the new sequence (not presently in [11])

$$
\overline{f f}(n), n \geq 1: 1,1,1,3,1,5,1,13,3,5,1,33,1,5,5,75,1,33,1,33,5,5,1,261, \ldots
$$

Another combinatorial interpretation of the numbers $\overline{f f}(n)$ is given in Section 4 .

\section{Application to systems complementing subsets}

Let $S=\left\{S_{1}, S_{2}, \ldots\right\}$ be a collection of nonempty sets of nonnegative integers. Then $S$ is called a system of complementing subsets for (or a complementing system of subsets of) $T \subset\{0,1,2, \ldots\}$ if every $t \in T$ can be represented uniquely as $t=s_{1}+s_{2}+\cdots$. with $s_{i} \in S_{i} \forall i$. This may also be expressed as $T=S_{1} \oplus S_{2} \oplus \cdots$, where $\oplus$ is the direct sum symbol. If there is a positive integer $k$ such that $T=S_{1} \oplus \cdots \oplus S_{k}$, then $S=\left\{S_{1}, \ldots, S_{k}\right\}$ is called a complementing $k$-tuple (for $T$ ). The set of all systems of complementing subsets for $T$ is denoted by $C S(T)$, and the set of complementing $k$-tuples by $C S(k, T)$.

In a fundamental paper de Bruijn [4] characterized the set $C S(\mathbb{N})$, where $\mathbb{N}=$ $\{0,1,2, \ldots\}$, and provided a full analysis of all complementing pairs for $\mathbb{N}$. The study of systems of complementing subsets for $\mathbb{N}_{n}=\{1,2, \ldots, n-1\}$, and hence, enumeration questions for systems of complementing subsets, were popularized by C. T. Long [8]. Among several other articles on the subject we mention [10] and [12].

The sequence $f f(n), n \geq 1$, begins as follows:

$$
1,1,1,2,1,3,1,5,2,3,1,11,1,3,3,15,1,11,1,11,3,3,1,45,2,3, \ldots
$$

This is identical with sequence A104725 in Sloane's database [11]: number of complementing systems of subsets of $\{0,1, \ldots, n-1\}$. 
There is a natural one-to-one correspondence between the sets $F F(n)$ and $C S\left(\mathbb{N}_{n}\right)$. For example $f f(4)=f(4)=2$ counts the complementing systems of $\{0,1,2,3\}$ namely $\{\{0,1,2,3\}\}$ and $\{\{0,1\},\{0,2\}\}$. The correspondence with $F F(4)$ is

$$
4_{1} \leftrightarrow\{\{0,1,2,3\}\}, \quad 2_{1} \cdot 2_{2} \leftrightarrow\{\{0,1\}\{0,2\}\} .
$$

The general bijection is obtained by associating each $g=g_{1} g_{2} \cdots g_{k} \in F(n)$ with the system

$$
\begin{aligned}
\left\{\left\{0, m_{0}, 2 m_{0}, \ldots,\left(g_{1}-1\right) m_{0}\right\},\left\{0, m_{1}, 2 m_{1} \ldots,\left(g_{2}-1\right) m_{1}\right\},\right. \\
\\
\left.\ldots,\left\{0, m_{k-1}, 2 m_{k-1} \ldots,\left(g_{k}-1\right) m_{k-1}\right\}\right\},
\end{aligned}
$$

where $m_{0}=1, m_{i}=g_{1} g_{2} \ldots g_{i}$, and each member of $X(n)$ with a certain system containing a subset different from the pattern $\{0, c, 2 c, \ldots, c r\}, c, r \geq 0$.

For example $g=\left(g_{1} g_{3}\right) g_{2} g_{4} g_{5} \cdots g_{k} \in X(n)$ maps to

$$
\begin{aligned}
& \left\{\left\{0, m_{0}, \ldots,\left(g_{1}-1\right) m_{0}\right\} \oplus\left\{0, m_{2}, 2 m_{2} \ldots,\left(g_{3}-1\right) m_{2}\right\},\left\{0, m_{1}, 2 m_{1} \ldots,\left(g_{2}-1\right) m_{1}\right\},\right. \\
& \left.\left\{0, m_{3}, 2 m_{3} \ldots,\left(g_{4}-1\right) m_{3}\right\}, \ldots,\left\{0, m_{k-1}, 2 m_{k-1} \ldots,\left(g_{k}-1\right) m_{k-1}\right\}\right\} .
\end{aligned}
$$

As an illustration, the implication

$$
2_{1} \cdot 2_{2} \cdot 3_{3} \in F(12,3) \Longrightarrow\left(2_{1} \cdot 3_{3}\right) \cdot 2_{2} \in X(12,2) \text { (proof of Theorem 2.6) }
$$

corresponds to the complementing systems implication

$$
\{\{0,1\},\{0,2\},\{0,4,8\}\} \Longrightarrow\{\{0,1\} \oplus\{0,4,8\},\{0,2\}\}=\{\{0,1,4,5,8,9\},\{0,2\}\} .
$$

Indeed the process of merging the atoms of $F(n, k)$ according to a partition of the label set of the atoms (Definition 2.4) corresponds to de Bruijn's original procedure of degeneration of complementing systems (see $[4,10]$ ). Thus if $P \in F F(n)$ maps to $U \in$ $C S\left(\mathbb{N}_{n}\right)$ under the bijection, the product of the atoms in each factor of $P$ corresponds to the cardinality of a component (member) of $U$. The fact that identical factors of $P$ bear different labels corresponds to the fact that components of $U$ with equal cardinalities contain different elements.

The full bijection between $F F(12)$ and $C S\left(\mathbb{N}_{12}\right)$ is shown in Table 1.

Finally, since the components of a complementing system are all distinct, we can isolate ordered complementing systems. A complementing system with $k$ components thus gives rise to $k$ ! ordered systems. For example $\{\{0,1\},\{0,2\},\{0,4,8\}\} \in C S\left(\mathbb{N}_{12}\right)$ gives the 6 systems

$$
\begin{aligned}
& \{\{0,1\},\{0,2\},\{0,4,8\}\},\{\{0,1\},\{0,4,8\},\{0,2\}\},\{\{0,2\},\{0,1\},\{0,4,8\}\}, \\
& \{\{0,2\},\{0,4,8\},\{0,1\}\},\{\{0,4,8\},\{0,1\},\{0,2\}\},\{\{0,4,8\},\{0,2\},\{0,1\}\} .
\end{aligned}
$$

In general the number $\overline{c s}(n)$ of ordered complementing systems of subsets of $\{1, \ldots, n-1\}$ is given by $\overline{c s}(n)=\sum_{k \geq 1} k ! c s(n, k)$, where $\operatorname{cs}(n, k)=\left|C S\left(k, \mathbb{N}_{n}\right)\right|$. Since the above bijection gives $\operatorname{cs}(n, k)=f f(n, k)$, we have,

$$
\overline{c s}(n)=\overline{f f}(n)=\sum_{j=1}^{\Omega(n)} f(n, j) \sum_{k=1}^{j} k ! S(j-1, k-1) .
$$




\begin{tabular}{c|c}
\hline Labeled Factorization of 12 & Complementing System of $\{0,1, \ldots, 11\}$ \\
\hline \hline $12_{1}$ & $\{\{0,1, \ldots, 11\}\}$ \\
\hline $2_{1} \cdot 6_{2}$ & $\{\{0,1\},\{0,2,4,6,8,10\}\}$ \\
\hline $6_{1} \cdot 2_{2}$ & $\{\{0,1,2,3,4,5\},\{0,6\}\}$ \\
\hline $3_{1} \cdot 4_{2}$ & $\{\{0,1,2\},\{0,3,6,9\}\}$ \\
\hline $4_{1} \cdot 3_{2}$ & $\{\{0,1,2,3\},\{0,2,4,8\}\}$ \\
\hline $2_{1} \cdot 2_{2} \cdot 3_{3}$ & $\{\{0,1\},\{0,2\},\{0,4,8\}\}$ \\
\hline $2_{1} \cdot 3_{2} \cdot 2_{3}$ & $\{\{0,1\},\{0,2,4\},\{0,6\}\}$ \\
\hline $3_{1} \cdot 2_{2} \cdot 2_{3}$ & $\{\{0,1,2\},\{0,3\},\{0,6\}\}$ \\
\hline$\left(2_{1} \cdot 3_{3}\right) \cdot 2_{2}$ & $\{\{0,1,4,5,8,9\},\{0,2\}\}$ \\
\hline$\left(2_{1} \cdot 2_{3}\right) \cdot 3_{2}$ & $\{\{0,1,6,7\},\{0,2,4\}\}$ \\
\hline$\left(3_{1} \cdot 2_{3}\right) \cdot 2_{2}$ & $\{\{0,1,2,6,7,8\},\{0,3\}\}$ \\
\hline
\end{tabular}

Table 1: The bijection between $F F(12)$ and $C S\left(\mathbb{N}_{12}\right)$.

\section{Application to generalized Bell numbers}

The enumeration of a subset of $X(n)$ leads to a class of generalized Bell numbers. This section and the next are devoted to the derivation and statement of their immediate properties.

The following definition is obtained from the proof of Theorem 2.1.

Definition 5.1. Let $d \mid n, d>1, q \in F F(d)$ and let $p \in F F(n)$ be derived from $q$ as described in the proof of Theorem 2.1. Then $p$ is called A-generated (by q) if it is obtained by appending $n / d$ at the end of $q$, and B-generated otherwise.

$A$ factorization of $n$ is called nested if it is (A or B) generated by a member of $X(d)$.

Thus a $p \in F F(n, k)$ is A-generated if and only if it is derived from a member of $F F(d, k-1)$. Equation (5) implies a decomposition of $f f(n)$ into the numbers of $A$ - and B-generated factorizations.

Denote the set of nested factorizations of $n$ by $X X(n)$. Then the number of non-nested factorizations of $n$ is given by

$$
f f(n)-x x(n)=f(n)+\sum_{\substack{d \mid n \\ d<n}} \sum_{k=1}^{\Omega(d)}(k-1) f(d, k)=1+\sum_{\substack{d \mid n \\ d<n}} \sum_{k=1}^{\Omega(d)} k f(d, k) .
$$

That is, besides the members of $F(n)$, non-nested factorizations include all (first-level) members of $X(n)$ which are B-generated by elements of $F(n)$. Consequently, using Equation (11), the number of nested factorizations of $n$ is given by

$$
x x(n)=\sum_{\substack{d \mid n \\ d<n}} \sum_{k=1}^{\Omega(d)} f(d, k)(B(k)-k) .
$$


The following alternative form is more complicated than (16), but it marks the appearance of a new ${ }^{2}$ class of generalized Bell numbers.

Theorem 5.2. The number of nested factorizations of $n$ is given by

$$
x x(n)=\sum_{d \mid n} \sum_{b=2}^{\Omega(d)} f(d, b) \sum_{j=2}^{\Omega(n / d)} f(n / d, j) B(j, b-1),
$$

where $B(n, b)$ is a generalized Bell number, the composite B-Bell number of order $b, b \geq 1$, defined below.

The composite B-Bell numbers $B(n, b)$ are defined in terms of the corresponding composite B-Stirling numbers of the second kind, $S(n, k, b)$, as follows

$$
B(0, b)=1, \quad B(n, b)=\sum_{k=1}^{n} S(n, k, b), \quad b \geq 1,
$$

and the $S(n, k, b)$ are given by

$$
\begin{gathered}
S(n, k, b)=S(n-1, k-1, b)+(k+b-1) S(n-1, k, b), \quad n \geq k, \\
S(0,0, b)=1, S(n, 1, b)=b^{n}, S(n, 0, b)=0, n>0 .
\end{gathered}
$$

The proof of Theorem 5.2 requires a formula on gozinta chains. Let $d, n$, be positive integers such that $d \mid n$. Define a $(d, n)$ gozinta chain of length $\ell$ as any increasing sequence of $\ell$ integers $d_{1}, d_{2}, \ldots, d_{\ell}$, satisfying $d=d_{1}, d_{\ell}=n$ and $d_{j-1} \mid d_{j}, 2 \leq j \leq \ell \leq \Omega(n)+1$.

Let $G(d, n)$ denote the set of $(d, n)$ gozinta chains. Then it is known (see [11, A034776]) that $|G(1, n)|=f(n)$. On dividing through the members of $G(d, n)$ by $d$ we obtain $G(1, n / d)$. Thus $|G(d, n)|=f(n / d)$.

It follows that the number $\ell_{k}(d, n)$ of $(d, n)$ gozinta chains of length $k$ is given by

$$
\ell_{k}(d, n)=f(n / d, k-1) .
$$

\section{Proof of Theorem 5.2}

Let $d \mid n, d>1$, and let $\ell_{d}+1, \ell_{d}>0$, be the length of a fixed $(d, n)$ gozinta chain, $d_{0}, d_{1}, \ldots, d_{\ell_{d}}\left(d=d_{0}, d_{\ell_{d}}=n\right)$.

Then clearly $X\left(d_{\ell_{d}}\right) \neq \varnothing$ whenever $\ell_{d} \geq 1$. In particular, $X X\left(d_{\ell_{d}}\right) \neq \varnothing$ if $\ell_{d} \geq 2$.

We follow the derivation of an element of $X X(n, k), k \geq b+1$, from some $q \in F(d, b+$ $1), b>0$, through the sets $X\left(d_{\ell}\right)$, where $X\left(d_{\ell}\right)$ or $X X\left(d_{\ell}\right)$ is assumed to be at level $\ell$, and $q$ is at level 0 .

Observe that $q=q_{0}$ B-generates $b$ elements $q_{1 i} \in X\left(d_{1}, b+1\right), 1 \leq i \leq b$, at level $\ell=1 \leq \ell_{d}$ (by inserting $\left.d_{1} / d\right)$. Each $q_{1 i}$ in turn generates a $q_{21} \in X X\left(d_{2}, b+2\right)$, and $b$ elements $q_{2 i} \in X X\left(d_{2}, b+1\right), 2 \leq i \leq b+1$, at level $\ell=2 \leq \ell_{d}$. Subsequently, this

\footnotetext{
2 "new" is used in the combinatorial sense since identical constructions of the numbers, up to linear translations, are known, see Section 6.
} 
process of generation of all-nested factorizations is repeated by each $q_{2 j}, 1 \leq j \leq b+b^{2}$, at level $\ell=3$, and so forth, until level $\ell=\ell_{d}$.

There are exactly $\ell$ different factorization lengths at level $\ell \geq 1$ namely $b+1, b+2, \ldots, b+\ell$. Assume that $S(\ell, b+k, b)=\left|X X\left(d_{\ell}, b+k\right)\right|, \ell \geq 2,1 \leq k \leq \ell$. Then we see that

$$
\begin{gathered}
S(\ell+1, b+k, b)=S(\ell, b+k-1, b)+(b+k-1) S(\ell, b+k, b), \\
S(\ell, b+1, b)=b^{\ell}, S(\ell, b, b)=0 .
\end{gathered}
$$

Indeed a $(b+k)$-factorization of $d_{\ell+1}$ can be uniquely A-generated by a $(b+k-1)$ factorization of $d_{\ell}$, or B-generated by a $(b+k)$-factorization of $d_{\ell}$ in $b+k-1$ ways (by inserting $n / d_{\ell}$ into $b+k-1$ possible factors excluding the factor whose last atom is labeled $b+k)$.

Note that since $k>0$, Equation (20) holds under the transformation $S(\ell, b+k, b) \mapsto$ $S(\ell, k, b)$.

Thus the number of elements of $X X(n, b+k)$ contributed by $q \in F(d, b+1)$ via all $(d, n)$ gozinta chains of length $j+1$ is

$$
\ell_{j+1}(d, n) S(j, k, b)=f(n / d, j) S(j, k, b), \quad 2 \leq j \leq \Omega(n / d),
$$

where the equality follows from (19). Therefore the total number for all $(d, n)$ gozinta chains and all factorization lengths is

$$
\sum_{k=1}^{j} \sum_{j=2}^{\Omega(n / d)} f(n / d, j) S(j, k, b) .
$$

Hence the number of elements of $X X(n)$ contributed by all members of $F(d, b+1)$ is

$$
\sum_{b=1}^{\Omega(d)-1} \sum_{j=2}^{\Omega(n / d)} f(d, b+1) f(n / d, j) \sum_{k=1}^{j} S(j, k, b) .
$$

Equation (20) is identical with the definition of the composite B-Stirling numbers of the second kind given in (18). The desired result follows from (21) and Equation (17).

Example 5.3. If $n=32$, then $f f(n)=52, x(n)=36, x x(n)=19$. The 19 nested factorizations are contributed by the following sets:

(1) $F(4,2)=\{2.2\}$ via the $(4,32)$ gozinta chains $(4,8,32),(4,16,32),(4,8,16,32)$, which respectively give 2,2,5 elements of $X X(32)$. For example, 2.2 and $(4,8,32)$ give $2_{1} \cdot 2_{2} \rightarrow\left(2_{1} \cdot 2_{3}\right) \cdot 2_{2} \rightarrow\left(2_{1} \cdot 2_{3}\right) \cdot\left(2_{2} \cdot 4_{4}\right),\left(2_{1} \cdot 2_{3}\right) \cdot 2_{2} \cdot 4_{4}$.

(2) $F(8,2) \cup F(8,3)=\{2.4,4.2,2.2 .2\}$ via the $(8,32)$ gozinta chain $(8,16,32)$. The factorizations give 2,2,6 elements of $X X(32)$ respectively. For example, 2.2 .2 and $(8,16,32)$ give

$$
2_{1} \cdot 2_{2} \cdot 2_{3} \rightarrow\left(2_{1} \cdot 2_{4}\right) \cdot 2_{2} \cdot 2_{3}, 2_{1} \cdot\left(2_{2} \cdot 2_{4}\right) \cdot 2_{3}
$$


and the latter factorizations give three elements each:

$$
\left(2_{1} \cdot 2_{4}\right) \cdot 2_{2} \cdot 2_{3} \rightarrow\left(2_{1} \cdot 2_{4}\right) \cdot\left(2_{2} \cdot 2_{5}\right) \cdot 2_{3}, \quad\left(2_{1} \cdot 2_{4}\right) \cdot 2_{2} \cdot\left(2_{3} \cdot 2_{5}\right), \quad\left(2_{1} \cdot 2_{4}\right) \cdot 2_{2} \cdot 2_{3} \cdot 2_{5},
$$

and so forth.

\begin{tabular}{c|c|c|c|c|c|c|c}
\hline$d$ & Factorization & $b$ & Gozinta & $\ell$ & $\mathrm{k}$ & $S(\ell, k+b, b)$ & $B(\ell, b)$ \\
\hline \hline$d_{0}$ & $g_{1} g_{2}$ & 1 & $\left(d_{0}, d_{1}\right)$ & 1 & 1 & 1 & 1 \\
\hline & & & $\left(d_{0}, d_{1}, d_{2}\right)$ & 2 & 1 & 1 & \\
& & & & 2 & 1 & 2 \\
\hline & & & $\left(d_{0}, d_{1}, d_{2}, d_{3}\right)$ & 3 & 1 & 1 & \\
& & & 2 & 3 & \\
& & & & 3 & 1 & 5 \\
\hline & & & $\left(d_{0}, d_{1}, d_{2}, d_{3}, d_{4}\right)$ & 4 & 1 & 1 & \\
& & & & 2 & 7 & \\
& & & & 3 & 6 & \\
& & & & 4 & 1 & 15 \\
\hline$d_{0}$ & $g_{1} g_{2} g_{3}$ & 2 & $\left(d_{0}, d_{1}\right)$ & 1 & 1 & 2 & 2 \\
\hline & & & $\left(d_{0}, d_{1}, d_{2}\right)$ & 2 & 1 & 4 & \\
& & & & 2 & 2 & 6 \\
\hline & & & $\left(d_{0}, d_{1}, d_{2}, d_{3}\right)$ & 3 & 1 & 8 & \\
& & & & 2 & 10 & \\
& & & & 3 & 2 & 20 \\
\hline & & & $\left(d_{0}, d_{1}, d_{2}, d_{3}, d_{4}\right)$ & 4 & 1 & 16 & \\
& & & & 2 & 38 & \\
& & & & 3 & 18 & \\
& & & & & 2 & 74 \\
\hline
\end{tabular}

Table 2: $X X(n)$-enumerating composite $B$-Stirling and $B$-Bell Numbers.

The connection with the composite B-Stirling numbers $S(\ell, k, b) \equiv S(\ell, b+k, b)$ is shown in Table 2 when the generating object has 2 or 3 factors. Recall that if $d \mid n$ and $F(d, b+1) \neq \emptyset$, then $S(\ell, k+b, b)$ counts the nested $(b+k)$-factorizations of $n$ contributed by an element of $F(d, b+1)$ through a $(d, n)$ gozinta chain $\left(d_{0}, d_{1}, \ldots, d_{\ell}\right), d=d_{0}, d_{\ell}=n, k=1,2, \ldots, \ell$.

We conclude this section with an interesting identity.

Theorem 5.4. Given positive integers $\ell, b, k, 1 \leq k \leq \ell, b>0$, then

$$
S(\ell, k+b, b)=b \sum_{j=1}^{k} S(\ell-j, k+b, b-1+j)
$$


Proof. Apply mathematical induction on the level $\ell$. The assertion holds for $\ell=1$ since $S(1, b+1, b)=b S(0, b+1, b)=b b^{0}=b$. Assuming that it holds for each of the levels $1,2, \ldots, \ell$, then it is easy to use Equation (20) and the hypothesis to prove $S(\ell+1, b+k, b)=\sum_{j=1}^{k} S(\ell+1-j, b+k, b-1+j)$. Hence the result.

Remark 5.5. Theorem 5.4 gives a recursive method of obtaining the number of nested $(b+k)$-factorizations at level $\ell$ from lower levels $\ell-1, \ell-2, \ldots$ More generally, if $Y(\ell, b)$ is the ordered multiset of factorization lengths $b+k$ occurring at level $\ell$, then (22) is equivalent to the assertion

$$
Y(\ell, b)=b \bigcup_{j=1}^{\ell} Y(\ell-j, b-1+j), \quad \text { where } r Y=(r y \mid y \in Y) .
$$

The relation is best visualized with tree diagrams. Figure 1 shows one branch of $Y(4,2)$ namely $\frac{1}{2} Y(4,2)=Y(3,2) \cup Y(2,3) \cup Y(1,4) \cup Y(0,5)$ (cf. $b=2$ in Table 2).

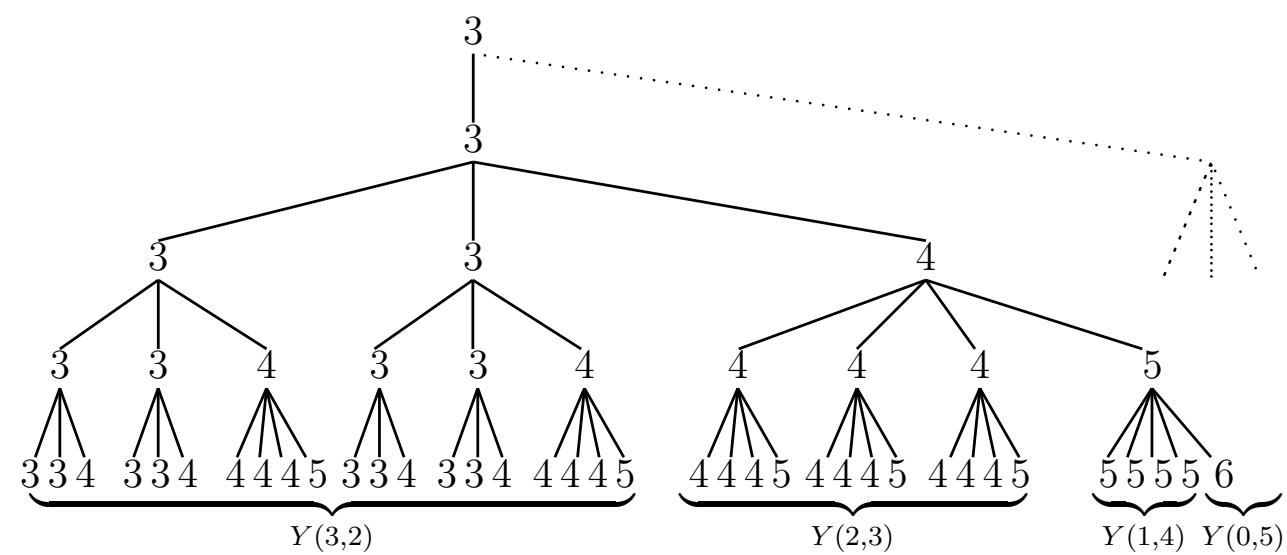

Figure 1: One branch of $Y(4,2)$ initially rooted at 3.

\section{The B-Stirling numbers}

The numbers $S(n, k, b)$ (see Equation $(18))$ are referred to as "composite" because each is a multiple of $b$. So we can divide by this common factor to obtain the reduced numbers.

$$
S_{b}(n, k)=\frac{1}{b} S(n, k, b), \quad B_{b}(0)=1, \quad B_{b}(n)=\sum_{k=1}^{n} S_{b}(n, k) .
$$

The following theorem is then immediate from (18) and (23).

Theorem 6.1. Let $n, k, b$, be positive integers such that $n \geq k>0$. Then

$$
S_{b}(n, k)=S_{b}(n-1, k-1)+(k+b-1) S_{b}(n-1, k),
$$




$$
S_{b}(n, 1)=b^{n-1}, S_{b}(n, 0)=\delta_{0 n},
$$

where $\delta_{i j}$ is the Kronecker delta.

Observe that $S_{1}(n, k)=S(n, k)$ and $B_{1}(n)=B(n)$.

The (reduced) generalized Stirling numbers $S_{b}(n, k)$ will be called simply B-Stirling numbers of the second kind of order $b$.

We state few striking properties of $S_{b}(n, k)$ which flow from Theorem (6.1) as corollaries below, omitting most standard-type results and proofs - these numbers are not entirely new (see comments at the end of this section).

Corollary 6.2. We have

(i)

$$
\sum_{n \geq 0} S_{b}(n, k) x^{n}=\frac{x^{k}}{(1-b x)(1-(b+1) x) \cdots(1-(k+b-1) x)} .
$$

(ii)

$$
S_{b}(n, k)=\sum_{j=1}^{k} \frac{(-1)^{k-j}(b+j-1)^{n-1}}{(j-1) !(k-j) !}, \quad 1 \leq k \leq n .
$$

(iii)

$$
S_{b+1}(n, k)=S_{b}(n, k)+k S_{b}(n, k+1) .
$$

Proof. Part $(i)$ is a routine consequence of Theorem 6.1, Part (ii) is obtained from $(i)$ using partial fractions (see [13] for similar ideas), and Part (iii) may be verified with (ii).

Corollary 6.3. We have

$$
\begin{gathered}
\sum_{n \geq 0} S_{b}(n+1, k) \frac{x^{n}}{n !}=\frac{e^{b x}\left(e^{x}-1\right)^{k-1}}{(k-1) !} . \\
S_{b}(n, k)=\sum_{r=0}^{n-1}\left(\begin{array}{c}
n-1 \\
r
\end{array}\right) S(r, k-1) b^{n-r-1} .
\end{gathered}
$$

Proof. Let $E_{b}(k, x)=\sum_{n \geq 0} S_{b}(n, k) x^{n} / n !$. Since $E_{1}(k, x)=\left(e^{x}-1\right)^{k} / k$ !, we have $\frac{d}{d x} E_{1}(k, x)=e^{x}\left(e^{x}-1\right)^{k-1} /(k-1)$ !, and since $E_{2}(k, x)=E_{1}(k, x)+k E_{1}(k+1, x)$ (Corollary (6.2)(iii)), we obtain $\frac{d}{d x} E_{2}(k, x)=e^{2 x}\left(e^{x}-1\right)^{k-1} /(k-1)$ !. So (24) follows by induction on $b$. Equation (25) is the result of extracting the coefficients of $x^{(n-1)} /(n-1)$ ! from both sides of $(24)$.

Lastly, Theorem 5.4 is restated as follows (apply $S(\ell, k+b, b) \rightarrow S(\ell, k, b) \rightarrow b S_{b}(\ell, k)$, see (23)): 
Corollary 6.4. Let $n, k, b$, be positive integers such that $n \geq k$. Then

$$
S_{b}(n, k)=\sum_{j=1}^{k}(b-1+j) S_{b-1+j}(n-j, k+1-j) .
$$

The case $b=1$ is noteworthy:

$$
S(n, k)=\sum_{j=1}^{k} j S_{j}(n-j, k+1-j) .
$$

Remark 6.5. Notice that (26) and (25) constitute a form of inverse relations.

Remark 6.6. Let $n, k, d$ be positive integers, $n \geq k$. Then it can be verified that the corresponding B-Stirling numbers of the first kind (unsigned) are the numbers $s_{b}(n, k)$, defined as follows.

$$
\begin{gathered}
s_{b}(n, k)=s_{b}(n-1, k-1)+(n+b-2) s_{b}(n-1, k), \\
s_{b}(n, 1)=\frac{(n+b-2) !}{(b-1) !}, s_{b}(n, 0)=0 .
\end{gathered}
$$

However, we are presently unable to associate $s_{b}(n, k)$ with any combinatorial interpretations.

Two authors have previously given different constructions which turn out to be equivalent to the B-Stirling numbers.

Carlitz [5] generalized ordinary partitions of $\{1, \ldots, n\}$ to $\lambda$-partitions, whereby some of the elements are distributed among $\lambda$ boxes, besides the blocks. The $\lambda$-partitions with $k$ blocks are enumerated by the weighted Stirling numbers, $R(n, k, \lambda)$ :

$$
\begin{gathered}
R(n+1, k, \lambda)=R(n, k-1, \lambda)+(k+\lambda) R(n, k, \lambda), \\
R(n, 0, \lambda)=\lambda^{n}, R(0, k, \lambda)=\delta_{0 k} .
\end{gathered}
$$

By comparing (27) and Theorem 6.1, we find that $R(n, k, \lambda)=S_{\lambda}(n+1, k+1)$.

The r-Stirling numbers, $\left\{\begin{array}{l}n \\ k\end{array}\right\}_{r}$, introduced by Broder [2], is founded on a simpler generalization of partitions. It answers the question: how many partitions, $B_{1} / \cdots / B_{k}$, of $\{1, \ldots, n\}$ have the property that $i \in B_{i}, i=1, \ldots, r$ ?

$$
\begin{gathered}
\left\{\begin{array}{l}
n \\
k
\end{array}\right\}_{r}=\left\{\begin{array}{l}
n-1 \\
k-1
\end{array}\right\}_{r}+k\left\{\begin{array}{c}
n-1 \\
k
\end{array}\right\}_{r}, \\
\left\{\begin{array}{l}
r \\
k
\end{array}\right\}_{r}=\delta_{r k},\left\{\begin{array}{l}
n \\
k
\end{array}\right\}_{r}=0, n<r .
\end{gathered}
$$

We easily deduce, from (28) and Theorem 6.1, that $\left\{\begin{array}{l}n \\ k\end{array}\right\}_{r}=S_{r}(n-r+1, k-r+1)$.

Apart from Corollary 6.4, which seems to be new, equivalent formulations of the results in this section, among several others, may be found in the papers of Carlitz and Broder. 


\section{Acknowledgments}

The author thanks Arnold Knopfmacher and David W. Wilson for helpful comments during the formative stages of this work.

\section{References}

[1] G. E. Andrews, The Theory of Partitions, Encyclopaedia of Mathematics and its Applications 2, Addison-Wesley, 1976.

[2] A. Z. Broder, The r-Stirling numbers, Discrete Math. 49 (1984), 241-259.

[3] R. A. Brualdi, Introductory Combinatorics, 2nd edition , Prentice Hall, 1992.

[4] N. G. de Bruijn, On number systems, Nieuw Arch. Wisk. 4 (1956), 15-17.

[5] L. Carlitz, Weighted Stirling numbers of the first and second kind, Parts I and II, Fibonacci Quart. 18 (1980) 147-162, 242-257.

[6] A. Knopfmacher, M.E. Mays, A Survey of factorization counting functions, Int. J. Number Theory 1 (2005), 653-581.

[7] A. Knopfmacher, M.E. Mays, Ordered and unordered factorizations of integers, Mathematica J. 10 (2006), 72-89.

[8] C. T. Long, Addition theorems for sets of integers, Pacific J. Math. 23 (1967), 107112.

[9] P. A. MacMahon, Memoir on the theory of the compositions of numbers, Philos. Trans. Roy. Soc. London (A) 184 (1893), 835-901.

[10] A.O. Munagi, k-Complementing subsets of nonnegative integers, Int. J. Math. Math. Sci. 2 (2005), 215-224.

[11] N. J. A. Sloane, (2006), The On-Line Encyclopedia of Integer Sequences, published electronically at http://www.research.att.com/ njas/sequences/.

[12] R. Tijdeman, Decomposition of the integers as a direct sum of two subsets, Number Theory (Paris, 1992-1993), London Math. Soc. Lecture Note Ser. 215, Cambridge University Press, 1995, pp. 261-276.

[13] H.S. Wilf, generatingfunctionology, Academic Press, Inc. 1994. 